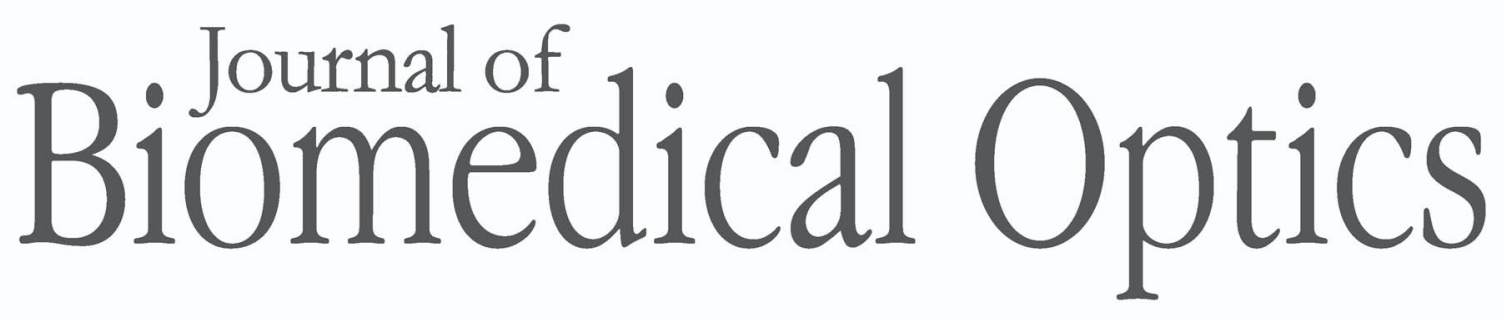

\title{
Injury depth control from combined wavelength and power tuning in scanned beam laser thermal therapy
}

Martin Villiger

Andrew Soroka

Guillermo J. Tearney

Brett E. Bouma

Benjamin J. Vakoc 


\title{
Injury depth control from combined wavelength and power tuning in scanned beam laser thermal therapy
}

\author{
Martin Villiger, Andrew Soroka, Guillermo J. Tearney, Brett E. Bouma, and Benjamin J. Vakoc \\ Harvard Medical School and Massachusetts General Hospital, Wellman Center for Photomedicine, 55 Fruit Street, \\ Boston, Massachusetts 02114
}

\begin{abstract}
Laser thermal therapy represents a possible method to treat premalignant epithelial lesions of the esophagus. Dynamically conforming the thermal injury profile to a specific lesion boundary is expected to improve the efficacy of such a treatment and avoid complications. In this work, we investigated wavelength tuning as a mechanism to achieve this aimed control over injury depth by using the strong variation of water absorption close to $1900 \mathrm{~nm}$. We developed a numerical model simulating in steps the photon propagation in the tissue, the diffusion of the absorbed heat, and the resulting tissue damage. The model was compared with experimental results on porcine esophageal specimens ex vivo and showed good agreement. Combined with power tuning, the wavelength agility in the range of 1860 to $1895 \mathrm{~nm}$ extends the injury range compared to a fixed wavelength source beyond $1 \mathrm{~mm}$, while at the same time improving control over shallow depths and avoiding vaporization at the tissue surface. The combination of two or three discrete wavelengths combined at variable ratios provides similar control, and may provide an improved strategy for the treatment of endothelial lesions. $\odot 2011$ Society of Photo-Optical Instrumentation Engineers (SPIE). [DOI: 10.1117/1.3647581]
\end{abstract}

Keywords: laser thermal therapy; laser injury; tissue response; damage model; injury depth control; Barrett's esophagus, thulium fiber laser.

Paper 11358RR received Jul. 8, 2011; revised manuscript received Sep. 16, 2011; accepted for publication Sep. 19, 2011; published online Oct. 26, 2011.

\section{Introduction}

Esophageal cancer caused 14,500 U.S. deaths in 2010 and its incidence is rising faster than that of all other malignancies in the western world. ${ }^{1}$ To improve these statistics, new techniques enabling earlier detection are needed in hand with strategies for early-stage intervention. Significant progress has been made toward improving diagnosis through optical imaging, for example, optical frequency domain imaging (OFDI). ${ }^{2}$ High resolution imaging of the esophagus using OFDI has been shown to provide high-speed, wide-field, and high-resolution $(\sim 10 \mu \mathrm{m})$ crosssectional imaging of the epithelial tissue microstructure using a balloon catheter deployment. ${ }^{3,4}$ The balloon acts to center an inner lumen within the tubular organ, and a side-looking imaging beam is rotated and translated within the inner lumen, scanning the imaging beam in a helical pattern, enabling wide-field imaging. Comprehensive in vivo imaging using this paradigm has been demonstrated in clinical studies. ${ }^{5-7}$

The past 5 years have additionally seen dramatic changes in the therapy of early-stage esophageal cancers, and in the management of Barrett's esophagus, a precursor lesion of esophageal adenocarcinoma. Radio-frequency (RF) ablation, which uses finely structured electrodes integrated into a balloon catheter to thermally coagulate the tissue at a superficial depth, has achieved unprecedented eradication rates of Barrett's esophagus. ${ }^{8}$ The ease of operation of RF ablation and the low incidence of complications may transform the standard-of-care treatment strategy for this disease. Instead of surveilling the patient and waiting

Address all correspondence to: Martin Villiger, Harvard Medical School and Massachusetts General Hospital, Wellman Center for Photomedicine, 40 Blossom Street - Bartlett Hall 704, Boston, Massachusetts 02114. Tel: (617) 643-6092; Fax: (617) 643-9208; E-mail: mvilliger@partners.org for Barrett's esophagus to develop into high grade dysplasia, treatment of low grade dysplasia and even nondysplastic epithelium is now envisioned. ${ }^{9}$ Recent studies established that in the short-term Barrett's esophagus is eradicated for a majority of the patients. ${ }^{10}$ However, despite repeated treatment sessions within 1 year, up to $23 \%$ of patients are left with metaplasia (11\% dysplasia). ${ }^{11}$ A possible explanation is the difficulty of applying the thermal therapy homogeneously over the irregular surface of the esophagus. The superficial application of the ablation without local control on the depth of the treatment also bears the risk of missing buried glands, which could reinitiate metaplasia at a later point in time. Patients are hence enrolled in post-treatment surveillance programs, which, together with the need for repeated treatment sessions, constrain the cost-efficacy of this approach. ${ }^{12}$

We have thus hypothesized that an image-guided therapy that combines improved imaging with spatially and depth controlled ablation therapy may dramatically improve the efficacy of this treatment and limit adverse complications and long term recurrence. In such an image-guided ablation device, a method for controlling the depth of the ablative injury is needed. In this work, we explore a mechanism to dynamically adjust the injury depth in laser thermal therapy (LTT), applied comprehensively in a beam scanning paradigm similar to that employed by the OFDI imaging system [Figs. 1(a)-1(c)]. Critical to this approach is the strong variation of the absorption coefficient of most biological tissue in the vicinity of the $1900 \mathrm{~nm}$ water absorption peak. Using combined wavelength and power tuning in this spectral region we demonstrate through both simulation and ex vivo studies that high fidelity depth control can be

1083-3668/2011/16(11)/118001/9/\$25.00 @ 2011 SPIE 
achieved. We further contrast the performance of two strategies of implementing depth controlled laser ablation: a continuous wavelength and power tunable therapy laser, and a combination of a set of individual power tunable fixed wavelength sources.

\section{Materials and Methods}

\subsection{Ex Vivo Studies}

We performed ex vivo laser ablation studies on porcine esophageal tissue samples harvested immediately after sacrifice and stored at $-80^{\circ} \mathrm{C}$. To generate scanned beam injuries, we placed the tissues on a metal fixture heated to $37^{\circ} \mathrm{C}$. The tissue samples were flattened and slightly compressed with a coverslip to ensure physical contact and as a proxy to balloon inflation. The epithelium was oriented against the glass coverslip. The dimensions of each tissue sample were approximately $3 \mathrm{~cm}$ $\times 4 \mathrm{~cm}$ along the top face and 3-mm thick after compression. The metal fixture was mounted to a motorized stage to provide fixed velocity translation at $v=2.2 \mathrm{~mm} \mathrm{~s}^{-1}$.

The collimated beam from a wavelength tunable thulium fiber laser (TFL) (IPG Photonics) was directed at the epithelium through the glass side. The TFL provided single-mode output at powers up to $1.2 \mathrm{~W}$ and wavelengths from 1860 to $1895 \mathrm{~nm}$. The collimated beam diameter was $1.3 \mathrm{~mm}$. Four linear injuries approximately $3 \mathrm{~cm}$ in length were generated in each tissue sample. The laser power and wavelength were held constant during exposure for each injury. A total of 32 laser settings were used, spanning the combination of four laser wavelengths (1860, $1870,1882.5$, and $1895 \mathrm{~nm})$ and 8 laser powers $(150,300,450$, $600,750,900,1050$, and $1200 \mathrm{~mW})$. The thermal therapy was monitored in real time with OFDI by focusing the imaging beam on the tissue surface in the center of the therapy beam.

After laser exposure, the metal fixture containing the compressed tissue was placed in an $-80^{\circ} \mathrm{C}$ freezer for $30 \mathrm{~min}$ to fix the conformation of the tissue. The frozen tissue was then removed and cut into smaller pieces for histological processing. The cuts were oriented perpendicular to the line injuries. Between 8 and 12 pieces were obtained from each tissue sample. Each piece was embedded in optimum cutting temperature compound for frozen processing and $10 \mu \mathrm{m}$ sections were cut. From each piece, a minimum of 2 slides were prepared using a nitroblue tetrazolium chloride (NBTC) histology stain specific for thermal injury, ${ }^{13,14}$ resulting in 16 to 22 histological sections for each generated injury. NBTC stains positive for the thermolabile enzyme lactate dehydrogenase (LDH). Loss of LDH activity ensues rapidly upon heat-induced cell damage and NBTC staining correlates with classical histology. ${ }^{15}$

For some pieces, additional sections were generated using NBTC staining with an eosin counterstain for enhanced structural visibility. Histology slides were digitized and the depth of each injury was read from the NBTC stained slides using ImageJ software. The injury depth was defined as the deepest point of unambiguous thermal injury.

\subsection{Laser Thermal Therapy Simulations}

To explore the impact of laser power and wavelength on injury depth and investigate important design parameters, we modeled the laser-tissue interaction and the coagulation of tissue. This simulation comprised three independent stages. The first stage used Monte Carlo methods to determine the three-dimensional energy absorption in the tissue as a function of the wavelength dependent absorption coefficient $\left(\mu_{\mathrm{a}}\right)$, the scattering coefficient $\left(\mu_{\mathrm{s}}\right)$, and the anisotropy $(\mathrm{g})$. The second stage used this absorption profile as the heat source in a three-dimensional, timedependent finite-difference modeling of the heat diffusion in the tissue. Finally, a third stage deduced from the time-dependent temperature profile the resulting tissue damage by modeling the protein denaturation with the Arrhenius equation as a first-order process. ${ }^{16}$

Monte Carlo modeling of light radiance in the tissue was carried out with the multilayered approach and source code of Wang et al., ${ }^{17}$ where our sample was modeled as a two-layer media. The first (superficial) layer described the $\sim 180 \mu \mathrm{m}$ thick coverslip used in the ex vivo studies $\left(\mu_{\mathrm{s}}=0, n=1.44\right.$, where $\mathrm{n}$ is the refractive index). The second layer described a homogeneous tissue $(n=1.38)$. To calculate the irradiance due to a $1.3 \mathrm{~mm}$ Gaussian beam, the result of the Monte Carlo simulation that corresponds to the photon distribution of an infinitely narrow photon beam in cylindrical coordinates and assuming circular symmetry, was interpolated on a three-dimensional grid and convolved with the Gaussian beam profile. The tissue optical properties were assumed to be independent of temperature. The scattering coefficient and anisotropy were modeled as wavelength-independent over the limited spectral range $(\Delta \lambda=50 \mathrm{~nm})$ of this study. The scattering coefficient was extrapolated following Collier et al. ${ }^{18}$ to $\mu_{\mathrm{s}}=21 \mathrm{~cm}^{-1}$, and an anisotropy of $g=0.9$ was assumed. The significant wavelength dependence of the absorption coefficient due to the nearby $1900 \mathrm{~nm}$ water absorption peak was included by using the absorption values for water reported by Wieliczka et al. ${ }^{19}$ [Fig. 1(d)]. The absorption of the tissue was obtained by multiplying the values for water by the water content of the tissue, which was estimated to be $70 \% .^{14}$

The diffusion of the heat deposited by the laser irradiation is governed by the laws of heat transfer. For the case of an isotropic medium with location dependent material properties the heat conduction is governed by:

$$
\nabla \cdot k \nabla T+Q=\rho C \frac{\delta T}{\delta t} .
$$

$k, \rho$, and $c$ are the thermal conductivity, the density, and the specific heat, respectively. $Q$ is the thermal source of the laser irradiation and was computed by the Monte Carlo simulation, and $T$ is the resulting temperature in function of space and time. The heat transfer was solved with a time-dependent finitedifference modeling in an explicit forward time model, using a control volume approach. ${ }^{20}$ The tissue and coverslip were described by a three-dimensional mesh of size $160 \times 160 \times 70$ $(x \times y \times z)$ where the $z$ dimension defines the depth into the tissue [Fig. 2(a)]. The mesh spacing was $\Delta x=60 \mu \mathrm{m}$ in all dimensions resulting in simulated tissue dimensions of $9.6 \mathrm{~mm}(x, y)$ $\times 4.2 \mathrm{~mm}(z)$. The temperature solution was advanced in time at $\Delta t=0.47 \mathrm{~ms}$ time steps. To include the effect of the scanned therapy beam, the temperature solution was shifted after each time step in the $+\mathrm{x}$ direction by $1.034 \mu \mathrm{m}(v \cdot \Delta t=2.2 \mathrm{~mm}$ $\left.\mathrm{s}^{-1} \cdot 0.47 \mathrm{~ms}\right)$. This simulation methodology revealed the temperature solution in a finite volume fixed to the location of the therapy beam, i.e., in a reference frame that was translating with 


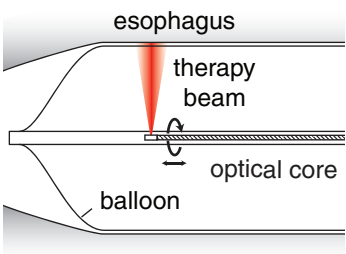

(a)

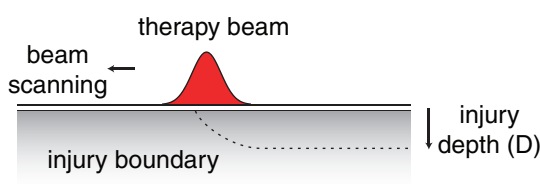

(c)

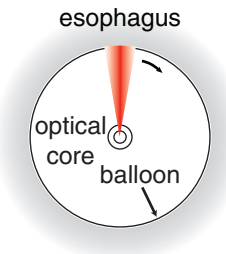

(b)

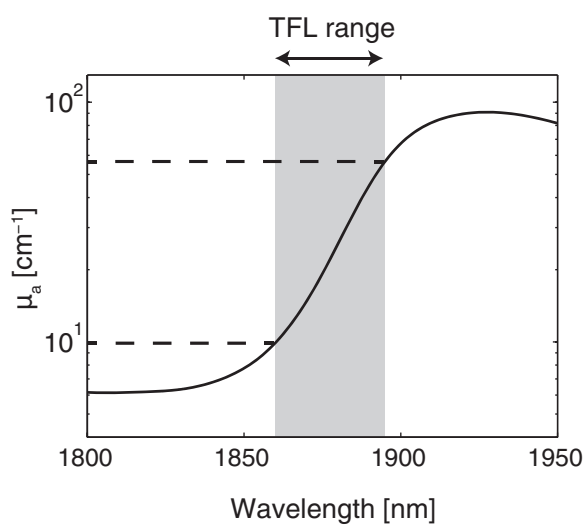

(d)

Fig. 1 Principle of wide-field beam scanning in the distal esophagus provided by balloon catheters in (a) longitudinal and (b) lateral cross section. The optical probe is rotated and slowly pulled back to sample large areas in a helical pattern. For OFDI imaging, the beam is focused at the surface to a $\sim 20$ to $30 \mu \mathrm{m}$ spot, whereas for LTT, a beam of several millimeters could be used. (c) At each scan location on the sample surface, the scanned therapy beam deposits a transient heat profile. Its cumulative effect and diffusion in the tissue determine the resulting injury depth. (d) Absorption coefficient of tissue assuming water content of $70 \%$, varying by nearly an order of magnitude when approaching the absorption peak at $1920 \mathrm{~nm}$.

the scanned beam rather than being fixed to the tissue. In the moving frame of the tissue, the spatial coordinate $x$ of the simulated volume becomes $x=v \cdot t$, revealing the temperature time trace at a fixed location. Because of the asymmetric temperature distribution in the moving frame, the therapy beam was located off-center in the $x$ dimension at $x=2.4 \mathrm{~mm}$, leaving $7.2 \mathrm{~mm}$ of length following the therapy beam [Figs. 2(a) and 2(b)].

The thermal conductivity and specific heat of esophageal tissue were estimated from Refs. 21 and 22 to be $k=0.5 \mathrm{~W} \mathrm{~m}^{-1}$ $\mathrm{K}^{-1}$ and $c=3.7 \mathrm{~J} \mathrm{~g}^{-1} \mathrm{~K}^{-1}$, with a density $\rho=1.05 \mathrm{~g} \mathrm{~cm}^{-3}$. The physical properties for the borosilicate coverslip were $k=1.13 \mathrm{~W} \mathrm{~m}^{-1} \mathrm{~K}^{-1}$ and $c=0.753 \mathrm{~J} \mathrm{~g}^{-1} \mathrm{~K}^{-1}$, with a density $\rho=2.17 \mathrm{~g} \mathrm{~cm}^{-3}$. The top surface $(z=0)$ was modeled as a convective boundary with a heat transfer coefficient $h=5 \mathrm{~W} \mathrm{~m}^{-2} \mathrm{~K}^{-1}$ and an air temperature of $37^{\circ} \mathrm{C}$. The tissue face preceding the therapy beam (i.e., at $x=0$ and not yet exposed to therapy light) was held fixed at $37^{\circ} \mathrm{C}$. Insulating boundary conditions were used on the remaining four faces. The tissue dimensions were chosen to prevent boundary effects from any but the top surface from significantly affecting the temperature solution. The time was iterated forward until the tissue was moved by a distance equivalent to 1.5 times the moving frame length. In this way the initial transient temperature profile was moved outside the reference frame, leaving the steady state solution.

The solution of the heat transfer equation defines the temperature history of the tissue as a function of depth and lateral position $(y)$ as it translates through the therapy beam exposure [Fig. 2(b)]. From this temporal temperature profile, the thermallyinduced coagulation injury was computed as a first order rate process, with the reaction rate constant given by the Arrhenius equation. The resulting damage integral in function of lateral position and depth is given as

$$
\Omega(z, y, \tau)=A \int_{0}^{\tau} \exp \left(-\frac{E_{a}}{R \cdot T(z, y, t)}\right) d t,
$$

where the parameters $A$ and $E_{a}$ are the frequency factor $\left(\mathrm{s}^{-1}\right)$ and activation energy for denaturation $\left(\mathrm{J} \mathrm{mol}^{-1}\right)$ for the tissue, and $R$ is the universal gas constant $\left(8.31 \mathrm{~J} \mathrm{~mol}^{-1} \mathrm{~K}^{-1}\right)$. The library of these parameters for specific tissues is growing but does not yet include values for esophageal mucosa. As a proxy, we have adapted those derived to describe laser-induced necrosis in swine skin, $A=2.88 \times 10^{98} \mathrm{~s}^{-1}$ and $E_{a}=6.3 \times 10^{5} \mathrm{~J} \mathrm{~mol}^{-1}$ as reported by Jacques, ${ }^{23}$ and originally evaluated by

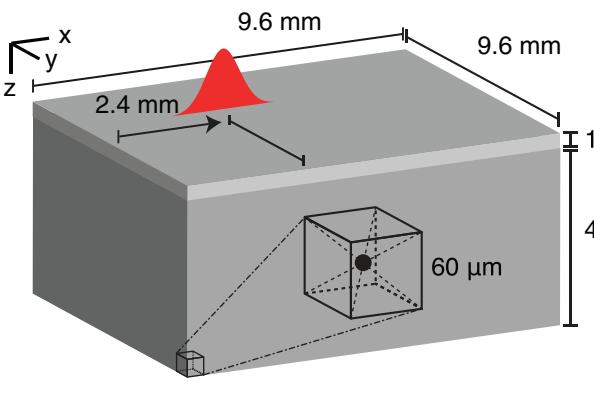

(a)

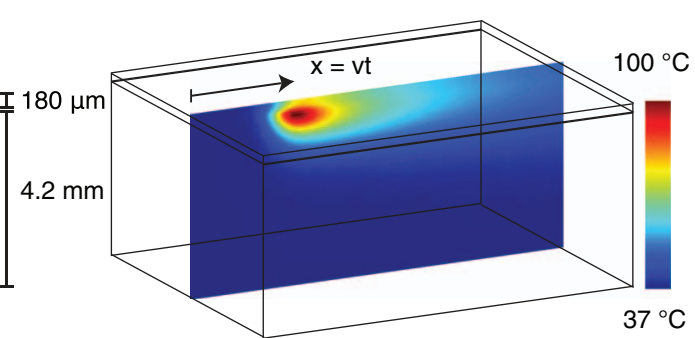

(b)

Fig. 2 Finite difference simulation of the heat transfer equation. (a) Dimensions and layout of the simulation. A control volume approach with centered node-points was used. Instead of scanning the beam, after each time iteration the tissue volume was moved along the x-coordinate. (b) Resulting spatiotemporal heat profile. 


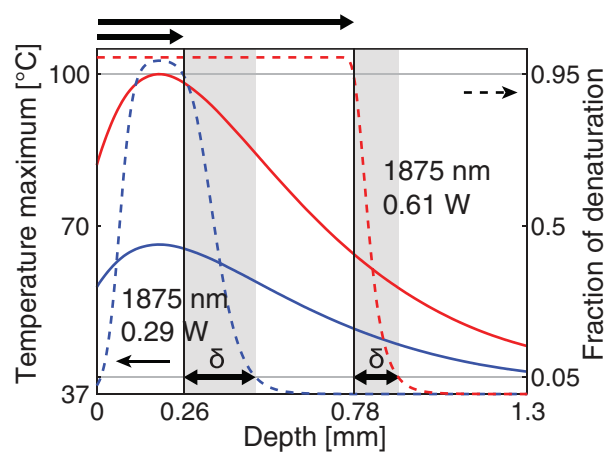

(a)

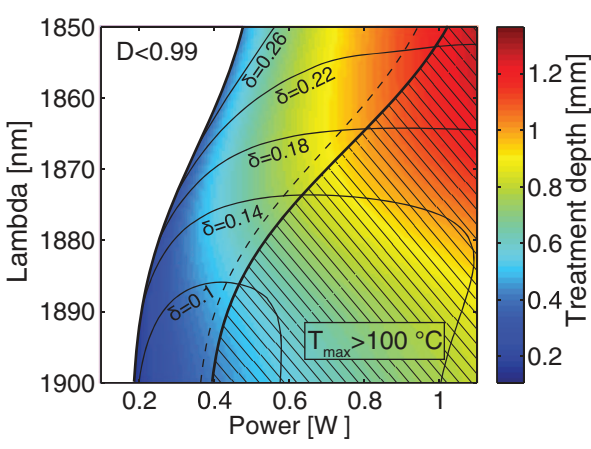

(c)

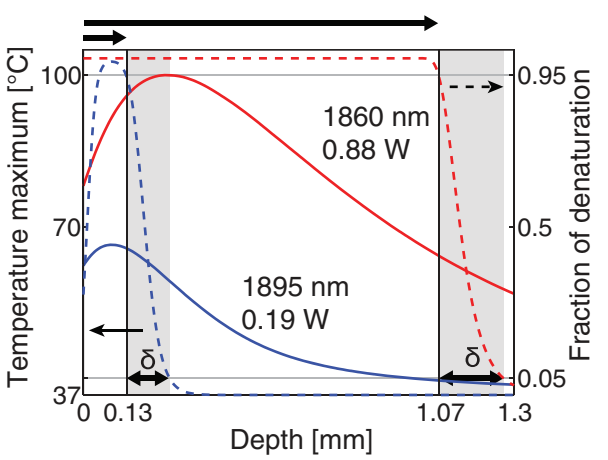

(b)

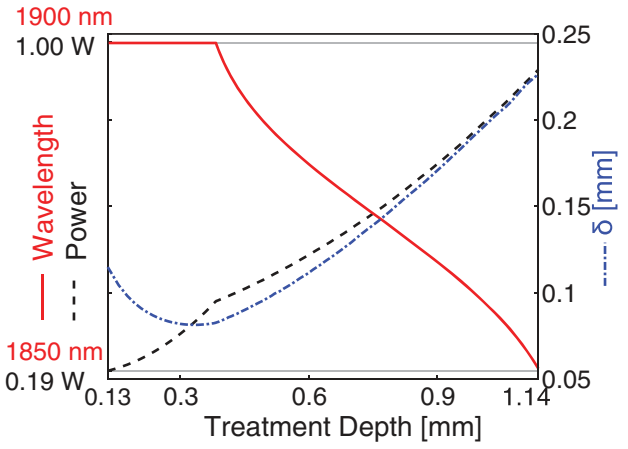

(d)

Fig. 3 Scanned beam thermal therapy. (a) Temperature maximum occurring during the therapy scan in function of depth at the center of the therapy beam, and resulting fractional tissue damage for a fixed wavelength of $1875 \mathrm{~nm}$ at two power settings. The 0.95 fractional damage was chosen as criterion for treatment depth, and the width of the transition region between 0.05 and 0.95 to quantify the margin $\delta$ of thermal collateral damage. The high power setting is limited by a $100^{\circ} \mathrm{C}$ threshold, whereas a fractional damage of 0.99 was used to determine the lower bound power setting. (b) The same upper and lower bound criteria, but applied to illumination at two distinct wavelengths increases the available treatment range. (c) Treatment depth in function of both wavelength and power. Only regions between the two thresholds are viable power-wavelength combinations. The contour lines indicate the variation of the margins $\delta$ in function of both parameters. (d) Following the upper threshold with a $5^{\circ} \mathrm{C}$ security margin, indicated by the dashed line in (c) defines the ideal wavelength-power combination for a given treatment depth. To access more shallow depths, the extreme wavelength of $1900 \mathrm{~nm}$ is power controlled, before starting the wavelength tuning.

Henriques. ${ }^{24}$ The integral extends from $t=0$, the beginning of the irradiation to $t=\tau$, the duration of the simulation window. The dimensions in the simulation were chosen to ensure that the reaction rate is $\ll 1$ at $t=\tau$ and no longer contributes to the overall damage integral. The fraction of thermally denatured proteins at a given spatial location is expressed as

$$
D(z, y)=1-\exp [-\Omega(z, y, \tau)] .
$$

For the assessment of the injury depth, the temperature profile and damage fraction were analyzed at midline of the therapy beam $(y=4.8 \mathrm{~mm})$. The tissue was assumed to be fully coagulated in regions where $D>0.95$.

\section{Results}

\subsection{Injury Depth Control in Single-Wavelength LTT is Limited by Vaporization and Accuracy Bounds}

To establish a baseline performance, the injury depth range achieved by power modulation of a fixed wavelength therapy beam was analyzed. The optical properties of the tissue at a specific wavelength define the effective penetration depth and energy distribution inside the tissue. Changing the laser power results in a linear scaling of this distribution. Because the finite- difference model is at equilibrium in the absence of any irradiation, the solution to the heat transfer equation likewise scales linearly with power around the initial temperature of $37^{\circ} \mathrm{C}$. Figure 3(a) displays the maximum temperature that occurred at each depth during exposure along the middle of the therapy beam for two different power settings and a wavelength of $1875 \mathrm{~nm}$. The shape of these temperature curves is defined by the tissue physical properties and the glass slide at the surface that acts as a heat sink and evacuates heat from regions adjacent to the surface. Whereas the temperature profile scales linearly with power, the damage integral does not. This reflects the tissue's susceptibility to temperatures above $\sim 64^{\circ} \mathrm{C}$, for which the activation energy for protein denaturation is exceeded. Assuming the tissue to be fully coagulated in regions where $D>0.95$ results in treatment depths of 0.26 and $0.78 \mathrm{~mm}$ for irradiation with 0.29 and $0.61 \mathrm{~W}$, respectively. The transitory region $0.95>$ $D>0.05$ was interpreted as a region of partial damage, where reversible damage such as edema and perivascular inflammation occur. In the interest of producing a clear treatment boundary, to reduce the risk of excessive collateral damage, and to ensure rapid healing and good tolerability of the treatment the margin, $\delta$, of this region should be minimized. ${ }^{25}$

At sufficiently high powers, the peak temperature at a superficial depth in the tissue reaches $100^{\circ} \mathrm{C}$, sufficient to induce 
vaporization. Although commonly used in the laser ablation of various tissue types, in the present setting vaporization is undesirable for two reasons. First, it results in an unpredictable and unstable injury zone. The removal and vaporization of superficial tissue likewise removes the landmarks necessary for guidance and control of the treatment depth. Second, vaporization induces dynamic modulations in the tissue properties that frustrate high resolution imaging. Thus, the vaporization threshold bounds the applicable power on the upper side and limits the achievable injury depth. Our model predicts a power threshold for vaporization of $0.61 \mathrm{~W}$ at a wavelength of $1875 \mathrm{~nm}$. For these parameters, the injury depth is limited to $0.78 \mathrm{~mm}$ [Fig. 3(a)].

As the power is reduced to drop the injury depth, several characteristics of the injury response are noteworthy. First, at sufficiently low powers, too little energy is deposited in the tissue to induce damage. This constitutes the most fundamental low-power/shallow-injury bound. The lower power setting of $0.29 \mathrm{~W}$ in Fig. 3(a) just reaches a fractional damage of 0.99 , and was used as criterion for the lower bound of the achievable injury depth. Due to the presence of the coverslip, the most superficial tissue is no longer completely coagulated in this configuration, but isolated from the spared tissue by a denatured layer. Also, at such low power, the slope of the temperature profile becomes very moderate and results in a larger margin $\delta$. This reduced temperature slope also has the effect that a slight change in power would induce significant variations in injury depth. Besides variation in power, the variability of tissue parameters that define thermal heating and practical limitations in precise delivery of laser light in clinical settings have a similar effect. A short margin $\delta$ is desirable to achieve accurate control over injury depths.

\subsection{Wavelength-Agile LTT Extends Injury Depth Control}

To broaden the treatable depth under the constraints of preventing vaporization and maintaining an acceptable injury margin $\delta$, an additional mechanism besides power tuning is needed to alter the depth-dependent energy deposition in the tissue. Varying the wavelength in order to tune the tissue absorption coefficient offers precisely this possibility. The absorption coefficient dominates the light distribution in the considered wavelength range. The absorption length $\mu_{\mathrm{a}}{ }^{-1}$ varies from $0.15 \mathrm{~mm}$ at $1900 \mathrm{~nm}$ to $1.28 \mathrm{~mm}$ at $1850 \mathrm{~nm}$, whereas the modeled transport mean free path is $\mu_{\mathrm{s}}^{-1} \cdot(1-\gamma)^{-1} \approx 5 \mathrm{~mm}$. Ignoring scattering, a variation in the absorption coefficient simply results in an altered exponential intensity decay along depth. The impact of this effect on injury depth is highlighted in Fig. 3(b) which shows the maximum temperature in the function of $\mathrm{z}$ and the resulting fractional tissue damage for the previously discussed bounds at the extreme wavelengths of our experimental laser source $(1860 \mathrm{~nm}$ : $\left.\mu_{\mathrm{a}}{ }^{-1}=1.00 \mathrm{~mm}, 1895 \mathrm{~nm}: \mu_{\mathrm{a}}{ }^{-1}=0.176 \mathrm{~mm}\right)$. At vaporization threshold, the injury depth is pushed to $1.07 \mathrm{~mm}$ and at the lower bound reduced to $0.13 \mathrm{~mm}$.

Figure 3(c) indicates the achievable injury depth for all possible wavelength-power combinations in the considered range, together with the contours of the resulting injury margin $\delta$. The vaporization threshold and the settings, which induce no damage to the tissue, define an area of viable wavelength-power combinations. As previously observed, with increasing power the treatment depth is extended. At the same time, the injury margin $\delta$ shrinks within the viable region. The eventual rise for power levels above the vaporization threshold has to be regarded as an artifact due to the temperature independence of the physical tissue properties employed by the model. At the least, this hypothesis breaks down by the induced phase change at vaporization. Close to the lower bound, the contour lines of the treatment margin become dense, which indicates again the strong sensitivity of the treatment depth and margin $\delta$ to variation of power close to the lower bound.

An isolated change of wavelength at maintained power has only little influence on the achieved treatment depth. If in Fig. 3(c) contour lines for constant treatment depth were drawn, they would be tangent to the wavelength axis for a specific wavelength. Indeed, this inflection point defines the wavelength with a minimized power requirement for a given treatment depth, which equals the absorption length in this case. For longer wavelengths, the energy is absorbed at more shallow depths, reducing the treatment depth. For shorter wavelengths, on the other hand, the energy is redistributed to larger depths, effectively diminishing the energy deposited at shallow depths, and inducing the observed recline in treatment depth.

However, the change of wavelength has an eminent influence on both the lower and upper power thresholds and in combination with power tuning considerably increases the range of treatment depths. Figure 3(d) indicates the ideal wavelength-power combination by first using the maximum wavelength of 1900 $\mathrm{nm}$ as a single wavelength source and then following the vaporization threshold at a $5^{\circ} \mathrm{C}$ security margin. The model estimates injury depths from $0.13 \mathrm{~mm}$ up to $1.14 \mathrm{~mm}$ with powers ranging from 0.19 to $1 \mathrm{~W}$. Together with an increase in treatment depth, an amplification of the margin $\delta$ in an approximately linear fashion comes in hand. Opposite to the increase of $\delta$ close to the lower bound, in the present case the boundary margin is however very robust as a function of power.

\subsection{Ex Vivo Measurements Confirm the Simulated Trend}

To validate the simulation results, we performed scanned beam therapy on porcine esophageal sections ex vivo at varying power and wavelength. The induced injury depth was assessed in histology using NBTC, which stains the thermolabile LDH and leaves thermally injured regions unstained.

Powers were selected at $150 \mathrm{~mW}$ intervals from $150 \mathrm{~mW}$ to $1.2 \mathrm{~W}$, and at four discrete wavelengths. Not all combinations induced a recognizable injury. The $150 \mathrm{~mW}$ power setting was below injury threshold for all four wavelengths, and at $300 \mathrm{~mW}$, only the high tissue absorption at the $1895 \mathrm{~nm}$ wavelength generated sufficient deposition of energy to leave a detectable injury in the NBTC stained sections. On the other hand, for the high power settings, the vaporization threshold reached, resulting at times in audible pops. The parallel monitoring of the exposure with OFDI allowed clear distinction between denaturation below vaporization threshold, which resulted in a continuous variation of the OFDI signal, ${ }^{26}$ and above threshold, in which case vaporization induced an abrupt disturbance in the signal.

Figures 4(a) and 4(b) show two examples of histology at the extreme injury depths, irradiating with just enough power 


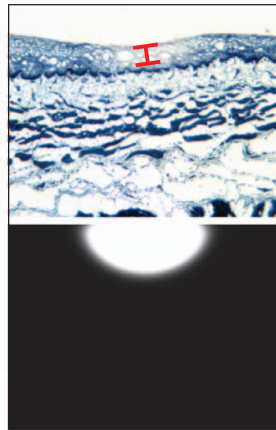

(a)

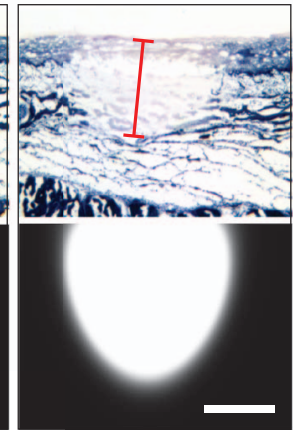

(b)

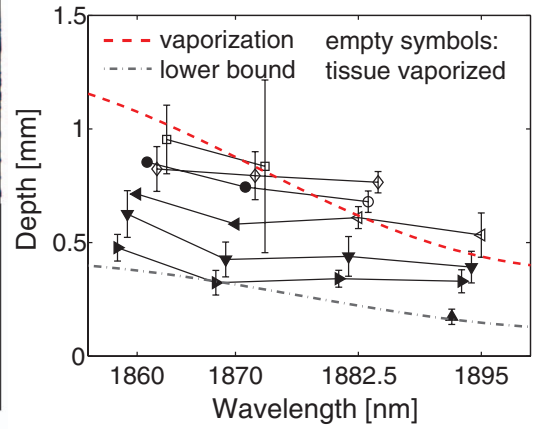

(c)

Fig. 4 Experimental validation of injury depth control by combined power and wavelength tuning. (a) and (b) Comparison of NBTC histology (top row, indicating the measured injury depth) with the fractional tissue damage according to the simulation for (a) $1895 \mathrm{~nm}$ and $0.30 \mathrm{~W}$ and (b) 1860 $\mathrm{nm}$ and $0.9 \mathrm{~W}$. (c) Measured injury depth (mean \pm SD, 16 to 22 individual sections for each data point) for different power-wavelength combinations. The spreading of the markers around the central wavelengths is for visualization purpose only.

to result in a thermal damage [Fig. 4(a)], and just below the vaporization threshold [Fig. 4(b)], respectively. The lower panel of Fig. 4 displays the lateral injury profile obtained from the simulation using matched wavelength-power settings. While the shape of the simulated injury matches well with histology, spatial dimensions are overestimated both laterally and in depth.

Figure 4(c) shows the experimentally measured injury depths for the various wavelength-power combinations. The empty symbols mark the settings, which resulted in vaporization at the surface of the tissue. The vaporization and injury thresholds predicted by the simulation are displayed in the same graph and exhibit a good match with the experimental data. The largest discrepancy is found at $1860 \mathrm{~nm}$, in which case vaporization was induced below the theoretically predicted bound. Also, the variability in the measured injury depths is significant for some points, whereas it remains small for others. This can be attributed to local variations and inhomogeneities in the tissue samples, a situation that is very likely to be encountered in a clinical setting.

Overall, the histological measurements of injury confirm the trend described by simulation, i.e., an extension of the accessible injury depth is achieved when wavelength is included as a control parameter relative to a fixed wavelength response.

\subsection{LTT With Combination of Discrete Power-Tunable Wavelengths Provides Similar Depth Control}

Dynamically conforming the thermal injury profile to a specific treatment plan or lesion boundary may best be facilitated by continuous and rapid tuning of both the laser power and wavelength. The implementation of a rapid wavelength tuned source is feasible but rather involved, however, whereas fixed wavelength fiber lasers have a far simpler architecture and are commercially available. As an alternative to continuous wavelength tuning, we investigated multiplexing of a discrete number of power-tunable fixed wavelength sources as a more practicable solution.

From Fig. 3(c) it is clear that selecting as little as two wavelengths with overlapping treatment bounds would cover nearly the entire depth range available with the wavelengthtunable source. However, the predicted injury margin $\delta$ would be severely compromised, and low power settings close to the lower bound would be required. Again, because of the linearity of the heat transfer equation, a simple mixing of wavelengths results in a linear combination of the simulated temperature profiles. Figure 5(a) displays the injury response to a mixing of the two extreme wavelengths at 1850 and $1900 \mathrm{~nm}$. The assembly of the ideal ratio-power combination following the $95^{\circ} \mathrm{C}$ threshold is presented in Fig. 5(b). The drawback of combining only the two extreme wavelengths is an increased injury margin as compared to the same treatment depth with a continuously tuned wavelength.

Conceptually, this performance could be significantly improved by adding a third wavelength at an optimized intermediate wavelength of $1876 \mathrm{~nm}$, in a consecutive binary mixing, as indicated in Figs. 5(c) and 5(d). For shallow treatment depths, the power was gradually transferred from the $1900 \mathrm{~nm}$ to the $1876 \mathrm{~nm}$ wavelength line. To induce injuries beyond the upper threshold of this discrete wavelength, it was then gradually mixed with the $1850 \mathrm{~nm}$ wavelength. In this way, the injury margin penalty compared to the wavelength tunable scenario was minimized to an acceptably low level.

\section{Discussion}

Wavelength tuning of the therapy beam in LTT in addition to power tuning was shown to effectively extend the achievable injury depth range in both simulation and empirical studies, offering a range from about $150 \mu \mathrm{m}$ to beyond $1 \mathrm{~mm}$. Based on the simulation results, two conceptual approaches for implementation in therapy systems were discussed. In the first, a single therapy source with continuous wavelength tuning was used, and both power and wavelength were modulated along a predefined path in the two-dimensional parameter space to control injury depth. In the second, a set of two or three fixed wavelength sources were multiplexed, and the relative power of each line was controlled, imitating the effect of a tunable wavelength. While far simpler from the point of view of technical implementation, this second approach only slightly compromised injury control compared to a tunable wavelength in terms of the margin of the injury boundary, and hence seems to be a viable concept for depth controlled LTT. 


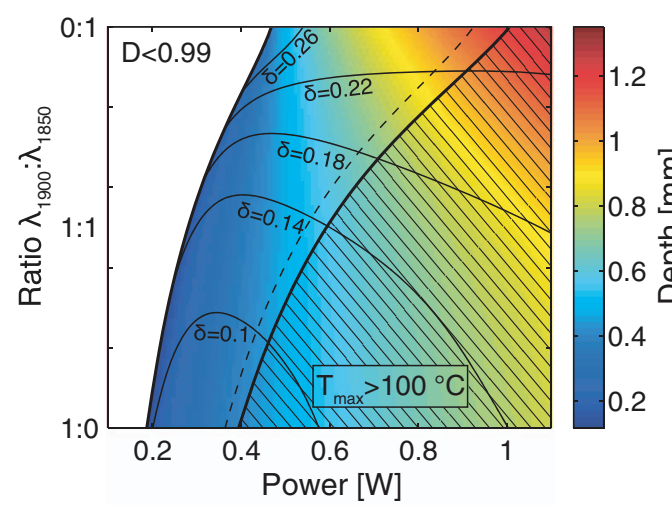

(a)

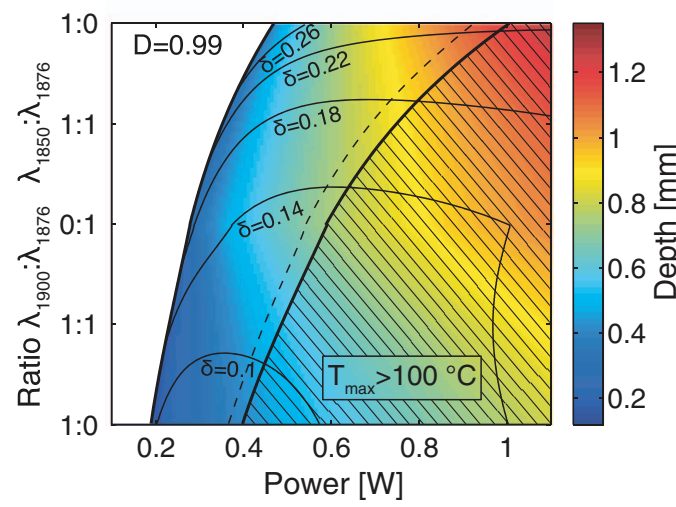

(c)

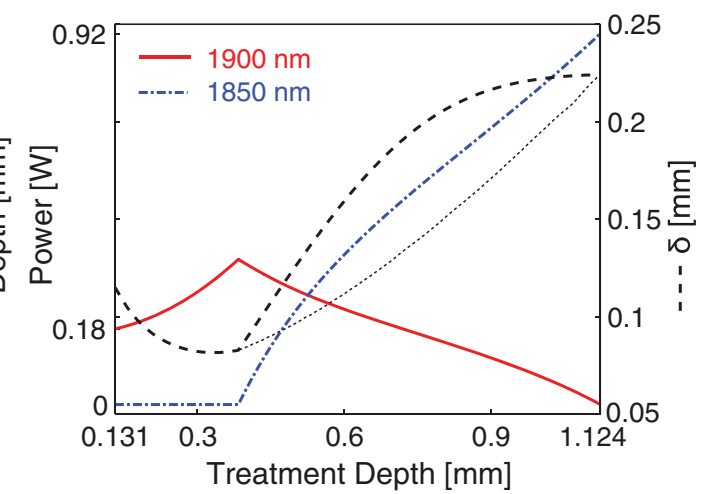

(b)

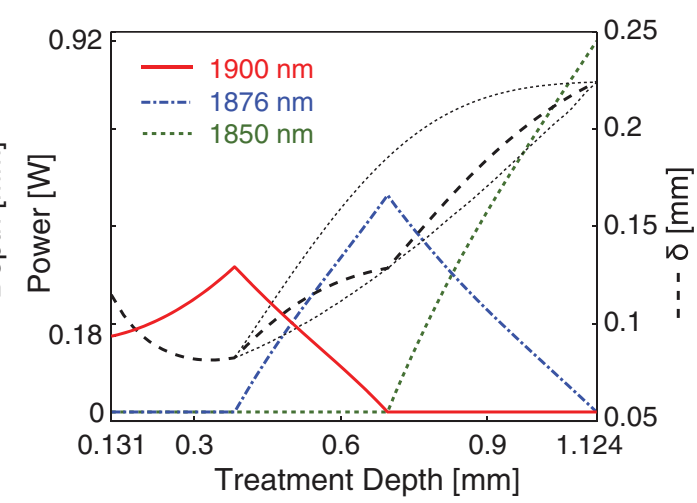

(d)

Fig. 5 Combining several discrete wavelengths results in a similar depth control. (a) Combining two wavelengths at 1850 nm and 1900 nm, and controlling their mixing ratio as well as the overall power produces a similar picture as the continuous wavelength scan displayed in Fig. 3 (c) [see legend of Fig. 3(c) for a detailed description]. (b) Resulting treatment depth and injury margin $\delta$ for the ideal mixing of the two wavelengths. The dotted line indicates the injury margin resulting from the continuous wavelength tuning. (c) Two consecutive binary combinations between wavelengths at 1900, 1876, and $1850 \mathrm{~nm}$. (d) Treatment depth and injury margin $\delta$ for the ideal parameter pairing. The dotted lines indicate the injury margins from the continuous wavelength tuning and from (b), respectively.

Controlling the injury depth by means of wavelength tuning is possible because of the absorption peak of water located around $1920 \mathrm{~nm}$. In the vicinity of this absorption peak, water is the dominant chromophore in biological tissue. The evaluated wavelength range offers injury depths from about $150 \mu \mathrm{m}$ to $1 \mathrm{~mm}$. Just beyond this range, the absorption coefficient peaks, and hence tuning beyond $1900 \mathrm{~nm}$ offers no benefit. Likewise, reducing the wavelengths below the evaluated range brings barely any gain in penetration depth. The absorption coefficient levels out [see Fig. 1(d)] and the light propagation in tissue becomes dominated by scattering, limiting the deposition of energy deeper inside the tissue.

Although the available treatment range might be sufficient to treat superficial endothelial lesions, a range of about $2 \mathrm{~mm}$ would be desirable to treat, for instance, the whole thickness of the squamous esophageal mucosa. Repeated treatment with intermediate removal of the coagulum could be considered to access larger depths. Another strategy to increase the therapy depth without reaching the vaporization threshold at the surface consists in actively cooling the top surface by forced convection. The presence of the coverslip in the simulations had a strong influence on the temperature profile. Acting as a heat sink, it evacuated heat from the topmost layer in the tissue, forcing the temperature profile to bend, and impacted strongly the vaporization threshold. The good match between experimental and simulated vaporization threshold in Fig. 4(c) was lost when running the simulations without coverslip and instead free convection at the tissue surface. Forced convection could serve to extend the injury depth, even to the point where the top surface no longer experiences thermal injury; this is a mechanism employed to treat only subsurface structures. ${ }^{27}$ Yet, such a configuration requires an excellent thermal contact between the heat sink and the tissue, which could be problematic in the envisioned clinical setting.

In this current study, a fractional tissue damage of 0.95 was used as the criterion for treatment depth, as used by others. ${ }^{22}$ Chen et al., ${ }^{28}$ on the other hand, defined the injury depth as the location where the damage integral $\Omega=1$, which is equivalent to a fractional damage of 0.63 . In their illustrative study, Chen et al. evaluate the thermal damage induced to pig skin in vivo, assessed $48 \mathrm{~h}$ after irradiation by histology, and match the injury depth predicted by the model with the depth of thrombosis in the dermis. They compare values for the frequency factor and the activation energy of the Arrhenius model from several sources. The best match is obtained for coefficients reported by Gaylor, ${ }^{29}$ which are similar to those employed by Khan et al., ${ }^{14}$ whereas all other sets of coefficients, including those of Henriques, ${ }^{24}$ used in our study, overestimated the treatment depth. Interestingly, 
applying Gaylor's coefficients in combination with Chen's criterion to our simulation aggravates the overestimation of our model. Whereas the cited studies considered a static treatment beam, the present work examines a scanned exposure beam, which introduces a smooth temporal envelope to the temperature profile. The resulting slower transition across the threshold temperature inducing thermal damage explains this seeming contradiction.

Chen et al. also analyzed tissue morphological changes and features beyond the induced thrombosis and necrosis, identifying a large zone of perivascular inflammation and edema. Arguing that this inflammatory response is a complex matter, beyond the purely physical effects of laser irradiation, they omitted a model prediction for the extent of this region. Despite this just argument, we employed the margin $\delta$ of the transition region between a fractional damage of 0.05 and 0.95 as a measure for this thermal collateral damage. It seems nevertheless reasonable to assume that an ideal temperature step profile induces a smaller irritation in the spared tissue than a slow gradient. Pulsed laser ablation has become a widely accepted method for this very reason. Short and high intensity laser pulses vaporize tissue very locally and nearly instantaneously, limiting the diffusion of heat to surrounding tissue. Only future studies monitoring tissue response to LTT in vivo will elucidate the influence of reversible thermal damage on the healing process and determine the tolerability of the proposed method. Besides the physiological motivation to reduce the transition region, a short $\delta$ with a steep temperature slope also reduces the uncertainty on the treatment depth and improves the accuracy of the depth control.

The aim of this work was to develop a numerical model for thermal tissue damage induced by a scanned therapy beam and to investigate the feasibility of wavelength tuning as a mechanism to control injury depth. Both the simulations and the experiments confirmed this approach. The development of more accurate models is certainly possible, but is questionable in view of the experimental setting, where most of the involved parameters are likely to change locally. Depending on these local tissue properties, the absolute mapping of power and wavelength to treatment depth will vary. The presented tuning mechanism, however, should prevail. In combination with OFDI, which can provide feedback on the progress of therapy in realtime ${ }^{26}$ true depth-controlled LTT could be achieved by continuously tuning the laser parameters until the image guided target depth is attained, rendering the therapy independent of tissue properties.

In conclusion, the present study discussed the use of wavelengths close to the water absorption peak at $1920 \mathrm{~nm}$ to control the thermally induced injury depth in epithelial tissue. We developed a numerical model in order to gain insight into the underlying physical mechanisms and corroborated the simulations with experimental results of LTT ex vivo. With the numerical model as a design tool at hand, we outlined two configurations for depth controllable LTT: a continuous power and wavelengthtunable laser source or, alternatively, a combination of two or three discrete and independently power controlled wavelength sources. Both approaches are anticipated to provide accurate control over the depth of thermally-induced damage to tissue, and may represent improved strategies for the treatment of endothelial lesions.

\section{Acknowledgments}

This work was supported by the National Institutes of Health (Grant Nos. R33CA125560 and K25CA127465).

\section{References}

1. A. C. Society, "Cancer Facts \& Figures," American Cancer Society, Atlanta: (2010).

2. S. Yun, G. Tearney, J. de Boer, N. Iftimia, and B. Bouma, "Highspeed optical frequency-domain imaging," Opt. Express 11, 2953-2963 (2003).

3. B. J. Vakoc, M. Shishko, S. H. Yun, W. Y. Oh, M. J. Suter, A. E. Desjardins, J. A. Evans, N. S. Nishioka, G. J. Tearney, and B. E. Bouma, "Comprehensive esophageal microscopy by using optical frequencydomain imaging (with video)," Gastrointest. Endosc. 65, 898-905 (2007).

4. W. Kang, H. Wang, Y. S. Pan, M. W. Jenkins, G. A. Isenberg, A. Chak, M. Atkinson, D. Agrawal, Z. L. Hu, and A. M. Rollins, "Endoscopically guided spectral-domain OCT with double-balloon catheters," Opt. Express 18, 17364-17372 (2010).

5. M. J. Suter, B. J. Vakoc, P. S. Yachimski, M. Shishkov, G. Y. Lauwers, M. Mino-Kenudson, B. E. Bouma, N. S. Nishioka, and G. J. Tearney, "Comprehensive microscopy of the esophagus in human patients with optical frequency domain imaging," Gastrointest. Endosc. 68, 745-753 (2008).

6. Y. Chen, A. D. Aguirre, P. L. Hsiung, S. Desai, P. R. Herz, M. Pedrosa, Q. Huang, M. Figueiredo, S. W. Huang, A. Koski, J. M. Schmitt, J. G. Fujimoto, and H. Mashimo, "Ultrahigh resolution optical coherence tomography of Barrett's esophagus: preliminary descriptive clinical study correlating images with histology," Endoscopy 39, 599-605 (2007).

7. M. J. Cobb, J. H. Hwang, M. P. Upton, Y. C. Chen, B. K. Oelschlager, D. E. Wood, M. B. Kimmey, and X. D. Li, "Imaging of subsquamous Barrett's epithelium with ultrahigh-resolution optical coherence tomography: a histologic correlation study," Gastrointest. Endosc. 71, 223-230 (2010).

8. N. J. Shaheen, P. Sharma, B. F. Overholt, H. C. Wolfsen, R. E. Sampliner, K. K. Wang, J. A. Galanko, M. P. Bronner, J. R. Goldblum, A. E. Bennett, B. A. Jobe, G. M. Eisen, M. B. Fennerty, J. G. Hunter, D. E. Fleischer, V. K. Sharma, R. H. Hawes, B. J. Hoffman, R. I. Rothstein, S. R. Gordon, H. Mashimo, K. J. Chang, V. R. Muthusamy, S. A. Edmundowicz, S. J. Spechler, A. A. Siddiqui, R. F. Souza, A. Infantolino, G. W. Falk, M. B. Kimmey, R. D. Madanick, A. Chak, and C. J. Lightdale, "Radiofrequency ablation in Barrett's esophagus with dysplasia," N. Engl. J. Med. 360, 2277-2288 (2009).

9. J. J. Bergman, "Radiofrequency ablation-great for some or justified for many?," N. Engl. J. Med. 360, 2353-2355 (2009).

10. T. Semlitsch, K. Jeitler, R. Schoefl, K. Horvath, N. Pignitter, F. Harnoncourt, and A. Siebenhofer, "A systematic review of the evidence for radiofrequency ablation for Barrett's esophagus," Surg. Endosc. 24, 2935-2943 (2010).

11. W. D. Lyday, F. S. Corbett, D. A. Kuperman, I. Kalvaria, P. G. Mavrelis, A. B. Shughoury, and R. E. Pruitt, "Radiofrequency ablation of Barrett's esophagus: outcomes of 429 patients from a multicenter community practice registry," Endoscopy 42, 272-278 (2010).

12. J. M. Inadomi, M. Somsouk, R. D. Madanick, J. P. Thomas, and N. J. Shaheen, "A cost-utility analysis of ablative therapy for Barrett's esophagus," Gastroenterology 136, 2101-2114 (2009).

13. R. A. Neumann, R. M. Knobler, F. Pieczkowski, and W. Gebhart, "Enzyme histochemical analysis of cell viability after argon laser-induced coagulation necrosis of the skin," J. Am. Acad. Dermatol. 25, 991-998 (1991).

14. M. H. Khan, R. K. Sink, D. Manstein, D. Eimerl, and R. R. Anderson, "Intradermally focused infrared laser pulses: Thermal effects at defined tissue depths," Lasers Surg. Med. 36, 270-280 (2005).

15. J. Hukki, J. Lipasti, M. Castren, P. Puolakkainen, and T. Schröder, "Lactate dehydrogenase in laser incisions: A comparative analysis of skin wounds made with steel scalpel, electrocautery, superpulsecontinuous wave mode carbon-dioxide lasers, and contact Nd:YAG laser," Lasers Surg. Med. 9, 589-594 (1989). 
16. J. Pearce and S. Thomsen, "Rate process analysis of thermal damage," in Optical-Thermal Response of Laser-Irradiated Tissue, pp. 561-608, A. Welch and M. van Gemert, Eds., (Plenum, New York 1995).

17. L. H. Wang, S. L. Jacques, and L. Q. Zheng, "MCML - Monte-Carlo modeling of light transport in multilayered tissues," Comput. Methods Programs Biomed. 47, 131-146 (1995).

18. T. Collier, M. Follen, A. Malpica, and R. Richards-Kortum, "Sources of scattering in cervical tissue: determination of the scattering coefficient by confocal microscopy," Appl. Opt. 44, 2072-2081 (2005).

19. D. M. Wieliczka, S. S. Weng, and M. R. Querry, "Wedge shaped cell for highly absorbent liquids-infrared optical-constants of water," Appl. Opt. 28, 1714-1719 (1989).

20. Y. Jaluria and K. E. Torrance, Computational Heat Transfer, Hemisphere Publishing Corp., Washington, D.C. (1986).

21. J. W. Valvano, J. R. Cochran, and K. R. Diller, "Thermal conductivity and diffusivity of biomaterials measured with self-heated thermistors," Int. J. Thermophys. 6, 301-311 (1985).

22. S. Puccini, N. K. Bar, M. Bublat, T. Kahn, and H. Busse, "Simulations of thermal tissue coagulation and their value for the planning and monitoring of laser-induced interstitial thermotherapy (LITT)," Magn. Reson. Med. 49, 351-362 (2003).
23. S. L. Jacques, "Ratio of entropy to enthalpy in thermal transitions in biological tissues," J. Biomed. Opt. 11, 041108 (2006).

24. F. C. Henriques, "Studies of thermal injury. V The predictability and the significance of thermally induced rate processes leading to irreversible epidermal injury," Arch. Pathol. 43, 489-502 (1947).

25. G. N. Paltauf and P. E. Dyer, "Photomechanical processes and effects in ablation," Chem. Rev. 103, 487-518 (2003).

26. B. J. Vakoc, G. J. Tearney, and B. E. Bouma, "Real-time microscopic visualization of tissue response to laser thermal therapy," J. Biomed. Opt. 12, 020501 (2007).

27. C. M. Cilip, N. J. Scott, S. R. Trammell, and N. M. Fried, "Noninvasive thermal coagulation of deep subsurface tissue structures using a laser probe with integrated contact cooling," in Conference Proceedings of the IEEE Eng. Med. Biol. Soc., Vol. 2008, pp. 3657-2660 (2008).

28. B. Chen, S. L. Thomsen, R. J. Thomas, J. Oliver, and A. J. Welch, "Histological and modeling study of skin thermal injury to $2.0 \mathrm{mu}$ m laser irradiation," Lasers Surg. Med. 40, 358-370 (2008).

29. D. C. Gaylor, "Physical mechanism of cellular injury in electrical trauma," Massachusetts Institute of Technology, 1989. 